\title{
Casimir energy due to inhomogeneous thin plates
}

\author{
C. D. Fosco and F. D. Mazzitelliø \\ Centro Atómico Bariloche, CONICET, Comisión Nacional de Energía Atómica, \\ R8402AGP Bariloche, Argentina \\ and Instituto Balseiro, Universidad Nacional de Cuyo, R8402AGP Bariloche, Argentina
}

(Received 29 December 2019; accepted 24 January 2020; published 18 February 2020)

\begin{abstract}
We study the Casimir energy due to a quantum real scalar field coupled to two planar, infinite, zerowidth, parallel mirrors with nonhomogeneous properties. These properties are represented, in the model we use, by scalar functions defined on each mirror's plane. Using the Gelfand-Yaglom's theorem, we construct a Lifshitz-like formula for the Casimir energy of such a system. Then we use it to evaluate the energy perturbatively, for the case of almost constant scalar functions, and also implementing a derivative expansion, under the assumption that the spatial dependence of the properties is sufficiently smooth. We point out that, in some particular cases, the Casimir interaction energy for nonplanar perfect mirrors can be reproduced by inhomogeneities on planar mirrors.
\end{abstract}

DOI: $10.1103 /$ PhysRevD.101.045012

\section{INTRODUCTION}

The evaluation of observables in the static Casimir effect presents many interesting challenges, both from the point of view of designing experiments capable of measuring its features with finer resolutions, as well as regarding the calculations involved in the prediction of those features [1]. In theoretical studies, the two main aspects usually considered in a system exhibiting the Casimir effect are the geometry of the mirrors and the properties of the media composing them.

In some special cases, for example when the mirrors are assumed to satisfy "perfect" boundary conditions, ${ }^{1}$ the ensuing simplifications allow one to study geometrical aspects in a simpler way. The derivative expansion (DE) is an approach that may be applied to the case of two smoothly curved surfaces, such that at each point their local separation is smaller than the curvature radii $[2,3]$. When the mirrors impose simple boundary conditions, the DE predicts the form of the corrections to the proximity force approximation (PFA), which is the leading term in the expansion. Indeed, the next-to-leading-order (NLO) term is completely fixed except for a single, global, dimensionless factor. That factor depends exclusively on the kind of

\footnotetext{
${ }^{1}$ Namely, boundary conditions which do not involve any parameter or function; typically, Dirichlet or Neumann for the case of a real scalar field, and perfect conductor conditions for the electromagnetic field.

Published by the American Physical Society under the terms of the Creative Commons Attribution 4.0 International license. Further distribution of this work must maintain attribution to the author(s) and the published article's title, journal citation, and DOI. Funded by SCOAP ${ }^{3}$.
}

boundary condition used, and it may be determined, once and for all, from a calculation which is perturbative in the departure from planar mirrors, to the second order in that departure. Note that the DE so obtained is not perturbative in the departure, yet it can be determined, for example, from the knowledge of that perturbative expansion.

The opposite, complementary case, would amount to systems with the simplest possible, yet nontrivial, geometry, like media having planar and parallel (inter)faces. Here, one can focus on the effects of the media inhomogeneities on the detailed properties of the Casimir force, in this case, its dependence on the distance between the mirrors. We are particularly interested in situations where the planar media are inhomogeneous at a macroscopic or mesoscopic level, that is, composed by regular patches or periodic arrays of homogeneous media. On general grounds, we expect the Casimir energy to exhibit a rich dependence on the inhomogeneities, similar to those that appear when considering perfect conductors with nontrivial geometries, as trench arrays and other periodic structures [4]. Such rich dependence was measured in good agreement with calculations for sinusoidally corrugated surfaces [5].

In this article, we consider the static Casimir effect for a special class of system, consisting of a quantum real scalar field in the presence of two zero-width, parallel and infinite plates. The latter are not assumed to be homogeneous; rather, each plate will be characterized, regarding its properties, by a real scalar potential defined on the plane. This can be considered as a toy model for the interaction of the electromagnetic field with a thin mirror characterized by position-dependent electromagnetic properties, and generalizes previous works for scalar fields in the presence of homogeneous thin plates, named $\delta$-potentials [6]. 
Under those assumptions about the system, and using the Gelfand-Yaglom's (GY) theorem [7], we shall obtain a Lifshitz-like formula [8], which yields the Casimir energy as a functional having as arguments the "scalar potentials," i.e., the two functions which characterize the plates [9]. We then use this generalized Lifshitz-formula to obtain more explicit expressions, making further assumptions about the potentials. We firstly consider the case of scalar potentials describing small departures from homogeneity, implementing a perturbative approach Casimir. We compare and relate the results to those corresponding to two Dirichlet mirrors with nonplanar geometries. On the other hand, if the space dependence of the properties is smooth, a DE for the energy becomes a useful tool. We use the perturbative results to obtain the usual PFA and its NLO for this particular system.

This paper is organized as follows: In Sec. II, we derive the would-be Lifshitz formula for the system. Then, in Sec. III, we present the expansion up to the second order in the departures from homogeneity, and discuss some particular limiting cases. In Sec. IV we apply the previous result to the calculation of the DE to the second order in derivatives. Section V contains our conclusions.

\section{LIFSHITZ FORMULA FOR NONHOMOGENEOUS PLATES}

We derive here an expression for the vacuum energy corresponding to a quantum real scalar field $\varphi(x)$ in $3+1$ dimensions, coupled to two nonhomogeneous zero-width, infinite, parallel plates. It may be regarded as a Lifshitz formula but for zero-width inhomogeneous plates, or even as a special case of the so-called TGTG formula [10] (here $\mathrm{T}$ stands for the Lippmann-Schwinger transition operator and $\mathrm{G}$ for the propagator).

The kind of model we deal with may be defined in terms of its Euclidean action $\mathcal{S}(\varphi)$, which is assumed to be of the form

$$
\mathcal{S}(\varphi)=\frac{1}{2} \int d^{4} x\left[(\partial \varphi(x))^{2}+V(\mathbf{x})(\varphi(x))^{2}\right]
$$

with spacetime coordinates $x=\left(x_{0}, x_{1}, x_{2}, x_{3}\right)$, and spatial coordinates $\mathbf{x}=\left(x_{1}, x_{2}, x_{3}\right)$. The "potential" $V$ accounts for the presence of two plates, denoted by $R$ and $L$, and located on the planes $x_{3}=\frac{l}{2}$ and $x_{3}=-\frac{l}{2}$, respectively. Specifically, that function is assumed to be of the form

$$
V(\mathbf{x})=\delta\left(x_{3}-\frac{l}{2}\right) V_{R}\left(\mathbf{x}_{\|}\right)+\delta\left(x_{3}+\frac{l}{2}\right) V_{L}\left(\mathbf{x}_{\|}\right),
$$

where $\mathbf{x}_{\|}=\left(x_{1}, x_{2}\right)$ are coordinates on the mirrors. We could also include a bulk potential $V_{\text {bulk }}\left(x_{3}, \mathbf{x}_{\|}\right)$between mirrors. This is a very interesting problem that raises mathematical and physical questions about the proper derivation of finite Casimir forces [11]. We plan to address this issue in a forthcoming paper. It is worth stressing that the potentials $V_{R, L}$ on the plates mimic inhomogeneities of the mirrors, that could be formed by a juxtaposition of homogeneous materials. Other kinds of situations, like frequency-dependent electromagnetic properties, would require, to be modeled, the presence of terms in the action which are nonlocal in time.

In the approach that we shall follow to derive the generalized Lifshitz formula, the vacuum energy will be obtained from the functional integral:

$$
\mathcal{Z}=\int \mathcal{D} \varphi e^{-\mathcal{S}(\varphi)} .
$$

Indeed, assuming the system to be defined within a "time interval": $-\frac{T}{2}<\tau<\frac{T}{2}$ and considering the $T \rightarrow \infty$ limit, since the potential has been assumed to be time independent, the leading behavior of the effective action $\Gamma$ is $\Gamma \sim T \times E, E$ being the vacuum energy. Thus, we may relate $E$ to a functional determinant, by using the formal result for $\Gamma$ which, ignoring irrelevant factors, is given by

$$
e^{-\Gamma}=\left[\operatorname{det}\left(-\partial^{2}+V\right)\right]^{-\frac{1}{2}},
$$

with $\partial^{2} \equiv \partial_{\mu} \partial_{\mu}, \mu=0,1, \ldots, d$.

Therefore

$E=\frac{1}{2} \int_{-\infty}^{+\infty} \frac{d k_{0}}{2 \pi} \log (\operatorname{det} \mathbb{K})=\frac{1}{2} \int_{-\infty}^{+\infty} \frac{d k_{0}}{2 \pi} \operatorname{Tr}(\log \mathbb{K})$,

where

$$
\mathbb{K}=-\nabla_{\mathbf{x}}^{2}+k_{0}^{2}+V(\mathbf{x}),
$$

and the determinant and trace are understood to be in the functional sense, for operators acting on functions of $\mathbf{x}$.

Our strategy to derive the generalized Lifshitz formula consists of "dimensionally reducing" the determinant appearing in (5). By this, we mean transforming it into one where the $x_{3}$ coordinate has been dealt with. Equivalently, at the level of the functional trace, it amounts to tracing out the $x_{3}$ coordinate, writing the result in terms of operators now acting on functions of $\mathbf{x}_{\|} \equiv\left(x_{1}, x_{2}\right)$ [rather than $\left.\mathbf{x}_{\|} \equiv\left(x_{1}, x_{2}\right)\right]$. That can be done by a suitable application of GY theorem, as follows. We first assume the system to be defined within a large "box" along the $x_{3}$ direction, namely, $-\frac{L}{2}<x_{3}<\frac{L}{2}$ where $L \gg l$, and we shall take the $L \rightarrow \infty$ limit at the end of the calculation. To proceed, we will need to assume some boundary conditions on $x_{3}= \pm \frac{L}{2}$. We shall, for the sake of simplicity, use Dirichlet conditions. ${ }^{2}$

We first rewrite the operator $\mathbb{K}$ introduced above in a way which makes it simpler to perform the dimensional reduction:

\footnotetext{
${ }^{2}$ The specific choice of boundary conditions has no bearing on the results, since we will take the $L \rightarrow \infty$ at the end of the calculation.
} 


$$
\mathbb{K}=-\partial_{3}^{2}+\mathbb{H}\left(x_{3}\right),
$$

where

$$
\mathbb{U}\left(x_{3}\right) \equiv-\nabla_{\|}^{2}+V_{x_{3}}\left(\mathbf{x}_{\|}\right)+k_{0}^{2},
$$

with

$$
V_{x_{3}}\left(\mathbf{x}_{\|}\right) \equiv V(\mathbf{x}), \quad \nabla_{\|} \equiv \nabla_{\mathbf{x}_{\|}} .
$$

The reason for this seemingly formal splitting is that $x_{3}$ is the variable about which the functional determinant of $\mathbb{K}$ will be reduced. To each value of $x_{3}$, we may associate an operator kernel along the $\mathbf{x}_{\|}$coordinates; indeed:

$$
\begin{aligned}
\left\langle\mathbf{x}_{\|}|\mathbb{K}| \mathbf{x}_{\|}^{\prime}\right\rangle & =K_{x_{3}}\left(\mathbf{x}_{\|}, \mathbf{x}_{\|}^{\prime}\right) \\
& =-\delta\left(\mathbf{x}_{\|}-\mathbf{x}_{\|}^{\prime}\right) \partial_{3}^{2}+H_{x_{3}}\left(\mathbf{x}_{\|}, \mathbf{x}_{\|}^{\prime}\right),
\end{aligned}
$$

where

$$
\begin{aligned}
H_{x_{3}}\left(\mathbf{x}_{\|}, \mathbf{x}_{\|}^{\prime}\right) & =\left\langle\mathbf{x}_{\|} \mathbb{W}\left(x_{3}\right) \mid \mathbf{x}_{\|}^{\prime}\right\rangle \\
& =-\nabla_{\mathbf{x}_{\|}}^{2} \delta\left(\mathbf{x}_{\|}-\mathbf{x}_{\|}^{\prime}\right)+\left[V_{x_{3}}\left(\mathbf{x}_{\|}\right)+k_{0}^{2}\right] \delta\left(\mathbf{x}_{\|}-\mathbf{x}_{\|}^{\prime}\right) .
\end{aligned}
$$

The corresponding result for the determinant is usually presented in terms of its ratio with a "reference" operator $\mathbb{K}_{0}$, which in our case we assume to correspond to a vanishing potential. Using this ratio instead of just $\mathbb{K}$ in (5) is indeed convenient, since it then produces the energy of the system taking as a reference the energy of the vacuum in the absence of the plates.

The ratio between determinants may be put in terms of two determinants of dimensionally reduced operators [12]:

$$
\frac{\operatorname{det} \mathbb{K}}{\operatorname{det} \mathbb{K}_{0}}=\frac{\operatorname{det} \psi\left(\frac{L_{d}}{2}\right)}{\operatorname{det} \psi_{0}\left(\frac{L_{d}}{2}\right)},
$$

where $\psi\left(x_{d}\right)$ and $\psi_{0}\left(x_{d}\right)$ are operatorial solutions to the homogeneous equations

$$
\mathbb{K} \psi\left(x_{d}\right)=0, \quad \mathbb{K}_{0} \psi_{0}\left(x_{d}\right)=0 .
$$

The matrix elements of these solutions may naturally be denoted by

$$
\left\langle\mathbf{x}_{\|}\left|\psi\left(x_{3}\right)\right| \mathbf{x}_{\|}^{\prime}\right\rangle=\psi_{x_{3}}\left(\mathbf{x}_{\|}, \mathbf{x}_{\|}^{\prime}\right)
$$

(and analogously for $\psi_{0}$ ). The initial conditions on the solutions to the homogeneous equations are

$$
\begin{gathered}
{\left.\left[\psi_{x_{3}}\left(\mathbf{x}_{\|}, \mathbf{x}_{\|}^{\prime}\right)\right]\right|_{x_{3}=-\frac{L}{2}}=0} \\
{\left.\left[\frac{\partial \psi_{x_{3}}}{\partial x_{3}}\left(\mathbf{x}_{\|}, \mathbf{x}_{\|}^{\prime}\right)\right]\right|_{x_{3}=-\frac{L_{3}}{2}}=\delta\left(\mathbf{x}_{\|}-\mathbf{x}_{\|}^{\prime}\right) .}
\end{gathered}
$$

We need a more explicit solution to Eq. (13); that can be done by first converting it to a first-order system, by introducing

$$
\Psi\left(x_{3}\right) \equiv\left(\begin{array}{c}
\psi\left(x_{3}\right) \\
\frac{\partial \psi}{\partial x_{3}}\left(x_{3}\right)
\end{array}\right) \equiv\left(\begin{array}{c}
\Psi_{1}\left(x_{3}\right) \\
\Psi_{2}\left(x_{3}\right)
\end{array}\right),
$$

which renders the original second-order equation into

$$
\frac{\partial \Psi}{\partial x_{3}}\left(x_{3}\right)=\mathcal{H}\left(x_{3}\right) \Psi\left(x_{3}\right)
$$

with

$$
\mathcal{H}\left(x_{3}\right)=\left(\begin{array}{cc}
0 & \mathbb{I} \\
\mathbb{H}\left(x_{3}\right) & 0
\end{array}\right),
$$

where $\mathbb{I}$ denotes the identity operator. Note that Eq. (17) is a Schroedinger-like equation in which the coordinate $x_{3}$ plays the role of time, and $\mathcal{H}$ the role of a "Hamiltonian." In terms of the evolution operator $\mathcal{U}$, we then have the solution:

$$
\Psi\left(x_{3}\right)=\mathcal{U}\left(x_{3},-\frac{L}{2}\right) \Psi\left(-\frac{L}{2}\right)
$$

with

$$
\mathcal{U}\left(x_{3}^{\prime \prime}, x_{3}^{\prime}\right) \equiv \mathcal{P} \exp \left[\int_{x_{3}^{\prime}}^{x_{3}^{\prime \prime}} d y_{3} \mathcal{H}\left(y_{3}\right)\right]\left(x_{3}^{\prime \prime} \geq x_{3}^{\prime}\right),
$$

with the path-ordering operator $\mathcal{P}$, which acts in the same way as the time-ordering operator, but with $x_{3}$ playing the role of the time.

Equipped with the solution for $\Psi$ just presented, we note that

$$
\left(\begin{array}{c}
\psi\left(\frac{L}{2}\right) \\
0
\end{array}\right)=\mathcal{U}\left(\frac{L}{2},-\frac{L}{2}\right)\left(\begin{array}{l}
0 \\
\mathbb{I}
\end{array}\right)
$$

or, using indices $A$ and $B$, which can assume the values 1 or 2 , to distinguish the 4 (operatorial) blocks in $\mathcal{U}\left(\frac{L}{2},-\frac{L}{2}\right)$ :

$$
\mathcal{U}\left(\frac{L}{2},-\frac{L}{2}\right) \equiv\left(\begin{array}{ll}
\mathcal{U}_{11}\left(\frac{L}{2},-\frac{L}{2}\right) & \mathcal{U}_{12}\left(\frac{L}{2},-\frac{L}{2}\right) \\
\mathcal{U}_{21}\left(\frac{L}{2},-\frac{L}{2}\right) & \mathcal{U}_{22}\left(\frac{L}{2},-\frac{L}{2}\right)
\end{array}\right),
$$

we see that 


$$
\frac{\operatorname{det} \mathbb{K}}{\operatorname{det} \mathbb{K}_{0}}=\frac{\operatorname{det} \mathcal{U}_{12}\left(\frac{L}{2},-\frac{L}{2}\right)}{\operatorname{det} \mathcal{U}_{12}^{(0)}\left(\frac{L}{2},-\frac{L}{2}\right)}
$$

This is a more explicit form of the reduction, where we still need to take the $L \rightarrow \infty$ limit. To do this we note that, since $V$ vanishes for $\left|x_{3}\right|>\frac{l}{2}$, we may write

$$
\begin{aligned}
\mathcal{U}\left(\frac{L}{2},-\frac{L}{2}\right)= & \lim _{\epsilon \rightarrow 0^{+}}\left[\mathcal{U}^{(0)}\left(\frac{L}{2}, \frac{l}{2}+\epsilon\right) \mathcal{U}\left(\frac{l}{2}+\epsilon,-\frac{l}{2}-\epsilon\right)\right. \\
& \left.\times \mathcal{U}^{(0)}\left(-\frac{l}{2}-\epsilon,-\frac{L}{2}\right)\right]
\end{aligned}
$$

where the $\epsilon \rightarrow 0^{+}$limit has been introduced, since the potential is discontinuous at $\pm \frac{l}{2}$.

The explicit form of $\mathcal{U}^{(0)}\left(x_{3}^{\prime}, x_{3}^{\prime \prime}\right)$ may be found by exponentiation, because it corresponds to an $x_{3}$-independent potential:

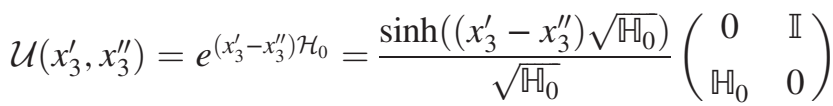

$$
\begin{aligned}
& +\cosh \left(\left(x_{3}^{\prime}-x_{3}^{\prime \prime}\right) \sqrt{\mathbb{I}_{0}}\right)\left(\begin{array}{ll}
\mathbb{I} & 0 \\
0 & \mathbb{I}
\end{array}\right) \text {. }
\end{aligned}
$$

Using this result, a rather lengthy but otherwise straightforward calculation shows that, when $L \rightarrow \infty$, the ratio between determinants may be written in terms of $\mu_{A B}$, a shorthand notation for the matrix elements

$$
\mu_{A B}=\lim _{\epsilon \rightarrow 0^{+}}\left[\mathcal{U}_{A B}\left(\frac{l}{2}+\epsilon,-\frac{l}{2}-\epsilon\right)\right]
$$

as follows:

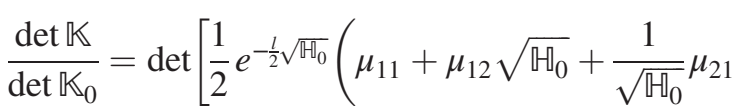

$$
\begin{aligned}
& \left.\left.+\frac{1}{\sqrt{\mathbb{\boxplus}_{0}}} \mu_{22} \sqrt{\mathbb{\boxplus}_{0}}\right) e^{-\frac{l}{2} \sqrt{\mathbb{\boxplus}_{0}}}\right] \text {. }
\end{aligned}
$$

The vacuum energy, referred to the vacuum in the absence of the plates, is then given by

$$
\begin{aligned}
& E=\frac{1}{2} \int_{-\infty}^{+\infty} \frac{d k_{0}}{2 \pi}\left\{\operatorname { l o g } \operatorname { d e t } \left[\frac { 1 } { 2 } \left(\mu_{11}+\mu_{12} \sqrt{\mathbb{Z}_{0}}+\frac{1}{\sqrt{\mathbb{G}_{0}}} \mu_{21}\right.\right.\right. \\
& \left.\left.\left.+\frac{1}{\sqrt{\mathbb{\mathbb { G } _ { 0 }}}} \mu_{22} \sqrt{\mathbb{\boxplus}_{0}}\right)\right]+\log \operatorname{det} e^{-l \sqrt{\mathbb{\boxplus}_{0}}}\right\} \text {. }
\end{aligned}
$$

In the previous expression, no use has been made yet of the precise form of the potential in the region occupied by the plates: this will determine the objects we have denoted by $\mu_{A B}$. Dealing with the effect of the $\delta$ functions, one can derive the explicit form

$$
\begin{aligned}
\left(\begin{array}{ll}
\mu_{11} & \mu_{12} \\
\mu_{21} & \mu_{22}
\end{array}\right)= & \left(\begin{array}{cc}
1 & 0 \\
V_{R} & 0
\end{array}\right)\left[\frac{\sinh \left(l \sqrt{\mathbb{U}_{0}}\right)}{\sqrt{\mathbb{G}_{0}}}\left(\begin{array}{cc}
0 & \mathbb{I} \\
\mathbb{\square} & 0
\end{array}\right)\right. \\
& \left.+\cosh \left(l \sqrt{\mathbb{G}_{0}}\right)\right]\left(\begin{array}{cc}
1 & 0 \\
V_{L} & 0
\end{array}\right),
\end{aligned}
$$

which, when inserted in (28), yields for the energy a result with the structure

$$
E=E_{L}+E_{R}+E_{I}
$$

with $E_{L}\left(E_{R}\right)$ denoting the self-energy of the $L(R)$ plate,

$$
E_{L, R}=\frac{1}{2} \int_{-\infty}^{+\infty} \frac{d k_{0}}{2 \pi} \operatorname{Tr} \log \left[\mathbb{I}+\frac{1}{2 \sqrt{\mathbb{\mathbb { Q }}}{ }_{0}} V_{L, R}\left(\mathbf{x}_{\|}\right)\right],
$$

where, we recall, $\mathbb{M}_{0}=-\nabla_{\|}^{2}+k_{0}^{2}$, and $E_{I}$ is the interaction energy, responsible for the Casimir force between mirrors. It is given by a generalized form of the Lifshitz formula,

$$
E_{I}=\frac{1}{2} \int_{-\infty}^{+\infty} \frac{d k_{0}}{2 \pi} \operatorname{Tr} \log \left[\mathbb{I}-e^{-l \sqrt{\mathbb{H}_{0}}} r_{R} e^{-l \sqrt{\mathbb{H}_{0}}} r_{L}\right]
$$

with the operatorial "reflection coefficients:"

$$
r_{L, R}=\left(2 \sqrt{\mathbb{M}_{0}}+V_{L, R}\right)^{-1} V_{L, R}
$$

Note that, except when the potentials are uniform, i.e., independent of $\mathbf{x}_{\|}$, these reflection coefficients involve noncommuting objects in their definitions, since $\mathbb{W}_{0}$ depends on the planar Laplacian operator. That makes it difficult to obtain more explicit expressions, unless extra assumptions are made.

\section{EXPANSION UP TO THE SECOND ORDER IN THE DEPARTURE FROM CONSTANT POTENTIALS}

Let us now expand Eq. (32), the general expression for the interaction part of the vacuum energy, up to the second order in the departure from constant functions:

$$
V_{L}\left(\mathbf{x}_{\|}\right)=v_{L}+\eta_{L}\left(\mathbf{x}_{\|}\right), \quad V_{R}\left(\mathbf{x}_{\|}\right)=v_{R}+\eta_{R}\left(\mathbf{x}_{\|}\right),
$$

where $v_{L}$ and $v_{R}$ are constants. We assume they are chosen in such a way that the spatial average of each departure vanishes. The expansion for the energy is then

$$
E_{I}=E^{(0)}+E^{(1)}+E^{(2)}+\ldots
$$

where

$$
\frac{E^{(0)}}{L^{2}}=\frac{1}{32 \pi^{2} l^{3}} \int_{0}^{\infty} d \rho \rho^{2} \log \left[1-\sigma_{L}(\rho) \sigma_{R}(\rho) e^{-\rho}\right],
$$

where $L^{2}$ is the area of the plates, and we have introduced 


$$
\sigma_{L, R}(\rho) \equiv \frac{x_{L, R}}{\rho+x_{L, R}}, \quad x_{L, R} \equiv l v_{L, R}
$$

Note that the Dirichlet limit is obtained as $\sigma_{L, R}(\rho) \rightarrow 1$. Equation (36) is easily obtained from Eq. (32) by setting $V_{L, R}=v_{L, R}$ in the reflection coefficients of Eq. (33).

The first-order term, on the other hand, vanishes. Indeed, it has the form

$$
E^{(1)}=\int_{\mathbf{x}_{\|}}\left[\left.\sum_{\alpha} \frac{\delta E}{\delta \eta_{\alpha}\left(\mathbf{x}_{\|}\right)}\right|_{\eta_{L} \equiv 0, \eta_{R} \equiv 0} \eta_{\alpha}\left(\mathbf{x}_{\|}\right)\right] .
$$

We have adopted above some conventions that we shall continue to use: a shorthand notation for the integration over $\mathbf{x}_{\|}$, and indices from the beginning of the Greek alphabet to denote each one of the two mirrors, namely, $\alpha=L, R$.

Now, the functional derivatives in (38) are evaluated for (simultaneously) vanishing $\eta_{L}$ and $\eta_{R}$, and are thus $\mathbf{x}_{\|}$ independent. Therefore,

$$
E^{(1)}=\left.\sum_{\alpha=L, R} \frac{\delta E}{\delta \eta_{\alpha}}\right|_{\eta_{L} \equiv 0, \eta_{R} \equiv 0} \int_{\mathbf{x}_{\|}} \eta_{\alpha}\left(\mathbf{x}_{\|}\right)=0 .
$$

The second-order term, $E^{(2)}$, is a quadratic form in the departures,

$$
E^{(2)}=\frac{1}{2} \sum_{\alpha, \beta} \int_{\mathbf{x}_{\|}, \mathbf{x}_{\|}^{\prime}} \eta_{\alpha}\left(\mathbf{x}_{\|}\right) \gamma_{\alpha \beta}\left(\mathbf{x}_{\|}-\mathbf{x}_{\|}^{\prime}\right) \eta_{\beta}\left(\mathbf{x}_{\|}^{\prime}\right),
$$

where the $\gamma_{\alpha \beta}$, being second functional derivatives at vanishing departures, can only depend on the differences between the arguments. Moreover, one can also show that they depend just on the modulus of $\mathbf{x}_{\|}-\mathbf{x}_{\|}^{\prime}$, since for constant potentials the plates are homogeneous and isotropic.

It is convenient to use, in what follows, Fourier transforms. We use a tilde on the Fourier transformed version of an object,

$$
\eta\left(\mathbf{x}_{\|}\right)=\int \frac{d^{2} \mathbf{k}_{\|}}{(2 \pi)^{2}} e^{i \mathbf{k}_{\|} \cdot \mathbf{x}_{\|}} \tilde{\eta}\left(\mathbf{k}_{\|}\right)
$$

and analogous conventions for Fourier transformation along the three coordinates $x_{0}, x_{1}, x_{2}\left(\equiv x_{\|}\right)$, on the space-time of the mirrors. As a technical remark, note that it is convenient, since the expressions become more symmetrical, to use space-time dependent departures, and to regard them as time independent at the end of the calculation.

Thus,

$$
E^{(2)}=\frac{1}{2} \sum_{\alpha, \beta} \int \frac{d^{2} \mathbf{k}_{\|}}{(2 \pi)^{2}} \tilde{\eta}_{\alpha}\left(-\mathbf{k}_{\|}\right) \tilde{\gamma}_{\alpha \beta}\left(\left|\mathbf{k}_{\|}\right|\right) \tilde{\eta}_{\beta}\left(\mathbf{k}_{\|}\right),
$$

where, having in mind the static limit mentioned above,

$$
\tilde{\gamma}_{\alpha \beta}\left(\left|\mathbf{k}_{\|}\right|\right)=\lim _{k_{0} \rightarrow 0}\left[\tilde{\gamma}_{\alpha \beta}\left(k_{\|}\right)\right] .
$$

To compute the $\tilde{\gamma}_{\alpha \beta}$, we need to expand the reflection coefficients in Eq. (33) up to second order in $\eta_{L, R}$, then insert the result in Eq. (32), and keep the quadratic terms. The expansion of the reflection coefficients, which has of course the same form for each plate, may be written as follows (we only write the one for the $L$ plate, the expansion for the other plate is obtained by replacing $L \rightarrow R$ below):

$$
r_{L}=r_{L}^{(0)}+r_{L}^{(1)}+r_{L}^{(2)}+\ldots
$$

where

$$
\begin{aligned}
& r_{L}^{(0)}=\left(2 \sqrt{\mathbb{\boxplus}_{0}}+v_{L}\right)^{-1} v_{L}, \\
& r_{L}^{(1)}=\frac{1}{v_{L}^{2}} r_{L}^{(0)} \eta_{L} r_{L}^{(0)} 2 \sqrt{\mathbb{G}_{0}}, \\
& r_{L}^{(2)}=-\frac{1}{v_{L}^{3}} r_{L}^{(0)} \eta_{L} r_{L}^{(0)} \eta_{L} r_{L}^{(0)} 2 \sqrt{\mathbb{I}_{0}} .
\end{aligned}
$$

The form of the order $n$ term, for any $n \geq 1$, is in fact

$$
r_{L}^{(n)}=(-1)^{n-1} \frac{1}{v_{L}^{n+1}}\left[r_{L}^{(0)} \eta_{L}\right]^{n} r_{L}^{(0)} 2 \sqrt{\mathbb{I}_{0}} .
$$

Inserting the previous expansion for each one of the plates to the second order, and expanding the logarithm accordingly, we may write down the different components of $\tilde{\gamma}_{\alpha \beta}$ more explicitly. The first ones we write down involve a departure in just one of the mirrors, to the second order:

$$
\begin{aligned}
\tilde{\gamma}_{L L}\left(k_{\|}\right)= & \frac{1}{2^{3} x_{L}^{3} l} \int \frac{d^{3} p_{\|}}{(2 \pi)^{3}} \frac{\left|p_{\|}\right| \sigma_{R}\left(p_{\|}\right) \sigma_{L}^{2}\left(p_{\|}\right) \sigma_{L}\left(p_{\|}+2 l k_{\|}\right)}{e^{\left|p_{\|}\right|}-\sigma_{L}\left(p_{\|}\right) \sigma_{R}\left(p_{\|}\right)} \\
& -\frac{1}{2^{4} x_{L}^{4} l} \int \frac{d^{3} p_{\|}}{(2 \pi)^{3}} \frac{\left|p_{\|}\right| \sigma_{R}\left(p_{\|}\right) \sigma_{L}^{2}\left(p_{\|}\right)}{e^{\left|p_{\|}\right|}-\sigma_{L}\left(p_{\|}\right) \sigma_{R}\left(p_{\|}\right)} \frac{\left|p_{\|}+2 l k_{\|}\right| \sigma_{R}\left(p_{\|}+2 l k_{\|}\right) \sigma_{L}^{2}\left(p_{\|}+2 l k_{\|}\right)}{e^{\left|p_{\|}+2 l k_{\|}\right|}-\sigma_{L}\left(p_{\|}+2 l k_{\|}\right) \sigma_{R}\left(p_{\|}+2 l k_{\|}\right)}, \\
\tilde{\gamma}_{R R}\left(k_{\|}\right)= & \frac{1}{2^{3} x_{R}^{3} l} \int \frac{d^{3} p_{\|}}{(2 \pi)^{3}} \frac{\left|p_{\|}\right| \sigma_{L}\left(p_{\|}\right) \sigma_{R}^{2}\left(p_{\|}\right) \sigma_{R}\left(p_{\|}+2 l k_{\|}\right)}{e^{\left|p_{\|}\right|}-\sigma_{R}\left(p_{\|}\right) \sigma_{L}\left(p_{\|}\right)} \\
& -\frac{1}{2^{4} x_{R}^{4} l} \int \frac{d^{3} p_{\|}}{(2 \pi)^{3}} \frac{\left|p_{\|}\right| \sigma_{L}\left(p_{\|}\right) \sigma_{R}^{2}\left(p_{\|}\right)}{e^{\left|p_{\|}\right|}-\sigma_{R}\left(p_{\|}\right) \sigma_{L}\left(p_{\|}\right)} \frac{\left|p_{\|}+2 l k_{\|}\right| \sigma_{L}\left(p_{\|}+2 l k_{\|}\right) \sigma_{R}^{2}\left(p_{\|}+2 l k_{\|}\right)}{e^{\left|p_{\|}+2 l k_{\|}\right|}-\sigma_{R}\left(p_{\|}+2 l k_{\|}\right) \sigma_{L}\left(p_{\|}+2 l k_{\|}\right)}
\end{aligned}
$$


and then we have the ones where each departure appears to the first order, which are symmetrical: $\tilde{\gamma}_{L R}=\tilde{\gamma}_{R L}$ :

$$
\begin{aligned}
\tilde{\gamma}_{L R}\left(k_{\|}\right)= & -\frac{1}{2^{4}\left(x_{L} x_{R}\right)^{2} l} \int \frac{d^{3} p_{\|}}{(2 \pi)^{3}} \frac{e^{\frac{1}{2}\left|p_{\|}\right|-\frac{1}{2}\left|p_{\|}+2 l k_{\|}\right|}}{e^{\left|p_{\|}\right|}-\sigma_{R}\left(p_{\|}\right) \sigma_{L}\left(p_{\|}\right)}\left|p_{\|}\right|\left|p_{\|}+2 l k_{\|}\right| \sigma_{R}\left(p_{\|}\right) \sigma_{L}\left(p_{\|}\right) \\
& \times \sigma_{R}\left(p_{\|}+2 l k_{\|}\right) \sigma_{L}\left(p_{\|}+2 l k_{\|}\right)\left[1+\frac{\sigma_{R}\left(p_{\|}+2 l k_{\|}\right) \sigma_{L}\left(p_{\|}+2 l k_{\|}\right)}{e^{\left|p_{\|}+2 l k_{\|}\right|}-\sigma_{R}\left(p_{\|}+2 l k_{\|}\right) \sigma_{L}\left(p_{\|}+2 l k_{\|}\right)}\right] .
\end{aligned}
$$

The term proportional to $\tilde{\gamma}_{L L}(R R)$ describes corrections to the Casimir energy produced by the inhomogeneities of the $L(R)$ plate, while the term proportional to $\tilde{\gamma}_{L R}$ is the relevant one for the computation of lateral forces and torques induced by the interaction between the inhomogeneities on both mirrors.

The rather complex expressions can be simplified for "quasi-Dirichlet" mirrors for which $x_{L, R} \gg 1$. In this limit we have $\sigma_{L, R}(\rho) \simeq 1-\rho / x_{L, R}$ and therefore the form factors $\tilde{\gamma}_{\alpha \beta}$ can be approximated by

$$
\begin{aligned}
x_{L}^{4} \tilde{\gamma}_{L L}\left(k_{\|}\right)= & x_{R}^{4} \tilde{\gamma}_{R R}\left(k_{\|}\right) \\
= & -\frac{1}{8 l} \int \frac{d^{3} p_{\|}}{(2 \pi)^{3}} \frac{\left|p_{\|}\right|\left|p_{\|}+2 l k_{\|}\right|}{\left(e^{\left|p_{\|}\right|}-1\right)\left(1-e^{-\left|p_{\|}+2 l k_{\|}\right|}\right)} \\
& \times\left(1-\frac{e^{-\left|p_{\|}+2 l k_{\|}\right|}}{2}\right)
\end{aligned}
$$

and

$$
\begin{aligned}
& \left(x_{L} x_{R}\right)^{2} \tilde{\gamma}_{L R}\left(k_{\|}\right) \\
& =-\frac{1}{16 l} \int \frac{d^{3} p_{\|}}{(2 \pi)^{3}} \frac{e^{\frac{1}{2}\left|p_{\|}\right|+\frac{1}{2}\left|p_{\|}+2 l k_{\|}\right|}\left|p_{\|}\right|\left|p_{\|}+2 l k_{\|}\right|}{\left(e^{\left|p_{\|}\right|}-1\right)\left(e^{\left|p_{\|}+2 l k_{\|}\right|}-1\right)},
\end{aligned}
$$

where we omitted a $k_{\|}$-independent term in $\tilde{\gamma}_{L L}$ and $\tilde{\gamma}_{R R}$. We can see that in this limit the $\gamma_{\alpha \beta}$ are all of the same order of magnitude if $x_{R} \simeq x_{L}$.

It is interesting to compare these results with the one for curved Dirichlet mirrors. For simplicity we will compare the interaction energy between Dirichlet mirrors (a curved mirror in front of a planar one) with that of a Dirichlet mirror $(\mathrm{R})$ in front of a quasi-Dirichlet planar mirror with inhomogeneities (L). The form factor for the former configuration, that we will denote here by $\tilde{\gamma}_{G}\left(k_{\|}\right)$is similar to $\tilde{\gamma}_{L L}[13]$ :

$\tilde{\gamma}_{G}\left(k_{\|}\right)=-\frac{1}{16 l^{5}} \int \frac{d^{3} p_{\|}}{(2 \pi)^{3}} \frac{\left|p_{\|}\right|\left|p_{\|}+2 l k_{\|}\right|}{\left(e^{\left|p_{\|}\right|}-1\right)\left(1-e^{-\left|p_{\|}+2 l k_{\|}\right|}\right)}$.

Note that the integrands of $\tilde{\gamma}_{L L}$ and $\tilde{\gamma}_{G}$ differ in a term proportional to $e^{-\left|p_{\|}+2 l k_{\|}\right|}$, and therefore they have the same behavior in the limit $\left|k_{\|}\right| l \gg 1$. Indeed, one can easily show that both are proportional to $\left|k_{\|}\right| l$ in this limit. Therefore, one can mimic a perfect mirror with nonplanar geometry with inhomogeneities on a planar mirror. The departure from the planar geometry of a Dirichlet mirror, $\eta_{G}\left(\mathbf{x}_{\|}\right)$, should be proportional to the departure from the constant potential $\eta_{L}\left(\mathbf{x}_{\|}\right)$. This is valid when the scale of variation of the geometry (and of the inhomogeneities) is much smaller that the distance between mirrors. The opposite limit will be studied in the next section.

\section{DERIVATIVE EXPANSION}

Let us now consider the case in which the inhomogeneities are smooth, that is, its scale of variation is much larger than $l$. We may extract from the results of the previous section the would-be zero order in the DE ("PFA") term. Indeed, from Eq. (36) we find

$$
E_{P F A}\left[V_{L}, V_{R}\right]=\int d^{2} \mathbf{x}_{\|} \mathcal{E}\left(l V_{L}\left(\mathbf{x}_{\|}\right), l V_{R}\left(\mathbf{x}_{\|}\right)\right),
$$

with

$\mathcal{E}\left(x_{L}, x_{R}\right)=\frac{1}{l^{3}} f\left(x_{L}, x_{R}\right)$,

$f\left(x_{L}, x_{R}\right)=\frac{1}{32 \pi^{2}} \int_{0}^{\infty} d \rho \rho^{2} \log \left(1-\frac{x_{L}}{\rho+x_{L}} \frac{x_{R}}{\rho+x_{R}} e^{-\rho}\right)$.

In Fig. 1 we plot the function $f\left(x_{L}, \infty\right)$, that describes the PFA to the interaction between a Dirichlet (R) and an

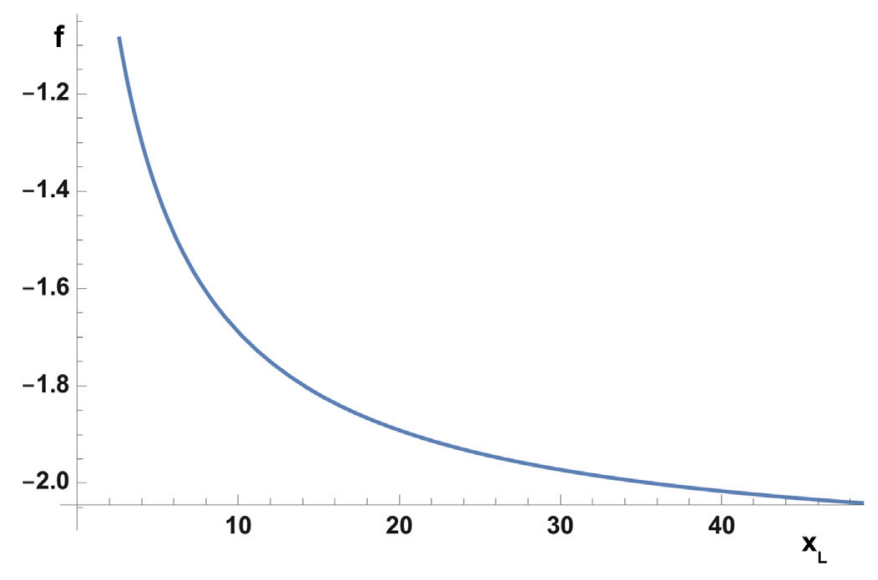

FIG. 1. The function $f\left(x_{L}, \infty\right)$ that describes the interaction between a Dirichlet mirror and an inhomogeneous mirror. It is negative and monotonically decreasing, vanishes linearly as $x_{L} \rightarrow 0$, and tends to $-\pi^{4} / 45$ as $x_{L} \rightarrow \infty$. 
inhomogeneous mirror (L). As expected, $f$ vanishes in the limit of transparent mirrors $\left(x_{L} \rightarrow 0\right)$, and tends to its Dirichlet value $\left(-\pi^{4} / 45\right)$ for $x_{L} \gg 1$. It interpolates monotonically between these two extreme cases.

To obtain the second-order term in a DE, which we denote by $E_{\mathrm{DE}}^{(2)}$, we follow our previous works $[2,3]$. We need to extract the term which is quadratic in $\mathbf{k}_{\|}$from each $\tilde{\gamma}_{\alpha \beta}$. Namely, for small $k_{\|}$:

$$
\tilde{\gamma}_{\alpha \beta}\left(k_{\|}\right)=c_{\alpha \beta} k_{\|}^{2}+\mathcal{O}\left(k_{\|}^{3}\right) .
$$

We have found

$$
c_{R R}=\frac{1}{48 \pi^{2} x_{R}^{3} v_{R}} \int_{0}^{\infty} d \rho \rho^{2}\left[[g(p)]^{2}-\frac{4 x_{L} x_{R}^{5}}{\left(\rho+x_{L}\right)\left(\rho+x_{R}\right)^{4}} \frac{1}{\left(\rho+x_{R}\right) e^{\rho}-\frac{x_{L} x_{R}}{\rho+x_{L}}}\right],
$$

where

$$
g(\rho)=-\frac{x_{L} x_{R}^{2}\left(e^{\rho}\left(\rho+x_{R}\right)\left(\rho^{3}+\rho^{2}\left(x_{L}+x_{R}+2\right)+\rho x_{L}\left(x_{R}+1\right)-x_{L} x_{R}\right)+x_{L} x_{R}^{2}\right)}{\left(\rho+x_{R}\right)^{2}\left(x_{L} x_{R}-e^{\rho}\left(\rho+x_{L}\right)\left(\rho+x_{R}\right)\right)^{2}}
$$

$c_{L L}=\left.c_{R R}\right|_{R \leftrightarrow L}$, and

$c_{L R}=\frac{l}{48 \pi^{2}\left(x_{L} x_{R}\right)^{2}} \int_{0}^{\infty} d \rho \rho^{2}\left\{\partial_{\rho}\left[\frac{e^{\frac{\rho}{2}} \rho \sigma_{L}(\rho) \sigma_{R}(\rho)}{e^{\rho}-\sigma_{L}(\rho) \sigma_{R}(\rho)}\right]\right\}^{2}$.

Therefore, going back to configuration space, the correction to the PFA reads

$$
E_{\mathrm{DE}}^{(2)}=-\frac{1}{2} \sum_{\alpha, \beta} \int d^{2} \mathbf{x}_{\|} c_{\alpha \beta}\left(l V_{L}, l V_{R}\right) \nabla V_{\alpha} \cdot \nabla V_{\beta}
$$

It is again instructive to analyze the case of quasiDirichlet mirrors. As before, we will consider the case in which the $\mathrm{R}$ mirror is perfect $\left(x_{R} \rightarrow \infty\right)$, and the $\mathrm{L}$ mirror is quasiperfect. Expanding the leading-order result for $x_{L} \gg 1$ we obtain

$$
E_{P F A}=-\frac{A \pi^{2}}{1440 l^{3}}\left(1-\frac{3}{l}\left\langle\frac{1}{V_{L}}\right\rangle\right)
$$

where $\langle\ldots\rangle$ denotes the mean value over the surface. On the other hand, in this limit

$$
c_{L L} \simeq \frac{k}{48 \pi^{2} l^{3} V_{L}^{4}},
$$

with

$k=\int_{0}^{\infty} d \rho \rho^{2}\left[\left(\frac{1+e^{\rho}(\rho-1)}{\left(e^{\rho}-1\right)^{2}}\right)^{2}-\frac{4}{e^{\rho}-1}\right] \simeq-9.14$

As a consequence, the NLO correction reads

$$
E_{\mathrm{DE}}^{(2)} \simeq \frac{9.14}{96 \pi^{2} l^{3}} \int d^{2} \mathbf{x}_{\|} \frac{\left(\nabla V_{L}\right)^{2}}{V_{L}^{4}}
$$

These results can be written in a simpler way by introducing a sort of "conductivity" $\rho_{L}=V_{L}^{-1}$ (note that $\rho_{L} \rightarrow 0$ for perfect mirrors)

$$
\frac{E_{\mathrm{DE}}}{A}=-\frac{\pi^{2}}{1440 l^{3}}\left(1-\frac{3}{l}\left\langle\rho_{L}\right\rangle\right)+\frac{9.14}{96 \pi^{2} l^{3}}\left\langle\left(\nabla \rho_{L}\right)^{2}\right\rangle .
$$

\section{CONCLUSIONS}

In this paper we evaluated the vacuum energy for a scalar field in the presence of two inhomogeneous thin plates. The interaction between the field and the plates is modeled by potentials localized on the plates. The calculation generalizes previous works on $\delta$-potentials and can be considered as a toy model for the interaction of the electromagnetic field with microstructured flat mirrors.

From a mathematical point of view, we used the GY theorem to compute the corresponding functional determinant, and arrived to a formal result for the vacuum energy, Eq. (32), that can be thought of as a generalized Lifshitz formula, in which the reflection coefficients are replaced by nonlocal operators that become local for homogeneous plates.

We have obtained explicit integral expressions for the vacuum energy in two different situations that involve either small or smooth inhomogeneities. For small inhomogeneities, we compared the result with the corresponding one for Dirichlet plates with nontrivial geometries, pointing out that when the scales of variation of the geometry and the inhomogeneity are much smaller than the distance between mirrors, geometry can be traded off by inhomogeneity. For smooth inhomogeneities, we used the 
DE as a expansion to obtain the NLO correction to the PFA approximation.

The formalism developed in this paper can be adapted to compute the vacuum energy in the electromagnetic case, including an inhomogeneous medium between plates. The calculation of Casimir-Polder forces on atoms near an inhomogeneous plate is also of interest. We plan to address these issues in a forthcoming work.

\section{ACKNOWLEDGMENTS}

This research was supported by ANPCyT, CONICET, and UNCuyo.
[1] P. W. Milonni, The Quantum Vacuum (Academic Press, San Diego, 1994); K. A. Milton, The Casimir Effect: Physical Manifestations of the Zero-Point Energy (World Scientific, Singapore, 2001); M. Bordag, G. L. Klimchitskaya, U. Mohideen, and V.M. Mostepanenko, Advances in the Casimir Effect (Oxford University Press, Oxford, 2009).

[2] C. D. Fosco, F. C. Lombardo, and F. D. Mazzitelli, Phys. Rev. D 84, 105031 (2011).

[3] G. Bimonte, T. Emig, and M. Kardar Appl, Appl. Phys. Lett. 100, 074110 (2012); G. Bimonte, T. Emig, R. L. Jaffe, and M. Kardar, Europhys. Lett. 97, 50001 (2012); C. D. Fosco, F. C. Lombardo, and F. D. Mazzitelli, Phys. Rev. A 89, 062120 (2014).

[4] A. Rodriguez, F. Capasso, and S. Johnson, Nat. Photonics 5, 211 (2011); F. Intravaia, S. Koev, I. Jung, A. A. Talin, P. S. Davids, R. S. Decca, V. A. Aksyuk, D. A. R. Dalvit, and D. López, Nat. Commun. 4, 2515 (2013).

[5] H.-C. Chiu, G. L. Klimchitskaya, V. N. Marachevsky, V. M. Mostepanenko, and U. Mohideen, Phys. Rev. B 80, 121402 (2009); 81, 115417 (2010); A. A. Banishev, J. Wagner,
T. Emig, R. Zandi, and U. Mohideen, Phys. Rev. Lett. 110, 250403 (2013).

[6] M. Bordag, D. Hennig, and D. Robaschik, J. Phys. A 25, 4483 (1992); K. A. Milton, J. Phys. A 37, 6391 (2004); Lect. Notes Phys. 834, 39 (2011).

[7] I. M. Gelfand and A. M. Yaglom, J. Math. Phys. (N.Y.) 1, 48 (1960).

[8] E. M. Lifshitz, Sov. Phys. JETP 2, 73 (1956).

[9] For a derivation of the usual Lifshitz formula using the GY theorem see C. Ccapa Ttira, C. D. Fosco, and F. D. Mazzitelli, J. Phys. A 44, 465403 (2011).

[10] O. Kenneth and I. Klich, Phys. Rev. B 78, 014103 (2008).

[11] For recent discussions see S. A. Fulling, T. E. Settlemyre, and K. A. Milton, Symmetry 10, 54 (2018); Y. Li, K. A. Milton, X. Guo, G. Kennedy, and S. A. Fulling, Phys. Rev. D 99, 125004 (2019).

[12] H. Kleinert, Path Integrals in Quantum Mechanics, Statistics, Polymer Physics, and Financial Markets (World Scientific, Singapore, 2004).

[13] See Eq. (28) in Ref. [2]. 